\title{
RESPUESTA EDUCATIVA A LA DIVERSIDAD DESDE LA PERSPECTIVA DEL PROFESORADO DE LA ESO: ESTUDIO EN LA COMUNIDAD AUTÓNOMA DE LA RIOJA*
}

\author{
Ma Asunción Jiménez Trens \\ Universidad de La Rioja \\ Ma Teresa Díaz Allué y \\ Rafael Carballo Santaolalla \\ Universidad Complutense de Madrid
}

\begin{abstract}
RESUMEN: La diversidad del alumnado de la ESO es una de las realidades más patentes, ricas y complejas de los centros de secundaria. Ofrecer adecuada respuesta educativa a todos los estudiantes compete, además de a otros estamentos, de modo ineludible a todo el profesorado. Analizar cómo perciben los profesores la diversidad y sus implicaciones, cómo valoran las medidas de atención a la diversidad, qué dificultades y apoyos encuentran en este cometido así como la formación que han recibido, es objeto de un amplio trabajo del que se presenta en este artículo una muy breve síntesis. Se ha realizado en la Comunidad Autónoma de La Rioja mediante un estudio de campo, por el método de encuesta y cuestionario como instrumento elaborado 'ad hoc', válido y fiable. Fueron sujetos de consulta todos los profesores de la ESO. Los datos han sido sometidos a los correspondientes análisis estadísticos que han permitido la obtención de conclusiones de las que se presenta un extracto.
\end{abstract}

ABSTRACT: The diversity of Secondary Education students is one of the most evident and at the same time richest and most complex realities in Secondary schools. Besides institutions and other governing organs, it is every teacher's responsibility -and one that cannot be neglected- to cater for all students' educational needs. It is the aim of this paper to analyze how teachers perceive such diversity with all its implications, and what difficulties and support they

* El contenido de este artículo tiene su origen básico en la tesis doctoral realizada por $\mathrm{M}^{\underline{a}}$ Asunción Jiménez Trens bajo la dirección de Maㅡ Teresa Díaz Allué y Rafael Carballo Santaolalla. 
find in this commitment, as well as the kind of training they have received in this respect. This is a broad aim that would require lenghthy and detailed treatment. Here we will just offer a very brief overview that discusses the results of work based on a field study that has been carried out in the Autonomous Community of La Rioja. The research methodology makes use of a survey and questionnaire developed as a valid and reliable 'ad hoc' instrument. All Secondary Education professionals were used as empirical subjects and the resulting data were put under statistical analysis. Some relevant conclusions are extracted and presented herein.

PALABRAS CLAVE: Diversidad. Educación Secundaria Obligatoria. Respuesta educativa a la diversidad.

KEYWORDS: diversity, compulsory Secondary Education, educational response to diversity

\section{INTRODUCCIÓN}

La Educación Secundaria Obligatoria (ESO) representa un importante, difícil y, a la vez, apasionante reto para sus responsables educativos. Se destina a un alumnado en la difícil edad de la adolescencia, en un clima socio-familiar que, en general, no favorece ni estimula el estudio, los hábitos ni el esfuerzo necesario para el trabajo intelectual, el aprendizaje y la formación integral. Además vivimos en una sociedad sumamente compleja, multicultural y multiétnica, con grandes diferencias sociales, caracterizada, entre otros rasgos, por la globalización, la sofisticación tecnológica en todos los ámbitos de la vida, la necesidad de la información y del conocimiento así como la de lograr el entendimiento, la paz y la convivencia entre personas, grupos, sociedades y países diferentes; sociedad a la que se denomina del conocimiento, del aprendizaje. En consecuencia, se observa que a las dificultades inherentes a esta etapa educativa se añaden las emanadas de la situación social que nos toca vivir y de las exigencias que todo ello comporta: formar a los jóvenes para que sean equilibrados, felices, solidarios y, al mismo tiempo, capaces de ser, en su día, profesionales competentes que actúen de acuerdo a los principios éticos.

El diseño del currículo de esta etapa, por su carácter obligatorio, ha de acometer una doble finalidad: terminal y propedéutica. Y, precisamente, esta característica confiere a esta etapa una particularidad que debe ser justamente interpretada tanto por el legislador como por los docentes implicados en su desarrollo e implementación. Esto, junto a otras variables de naturaleza social y personal, está teniendo unas evidentes implicaciones en la vida de los centros de Secundaria. Probablemente una de las más notables y significativas sea el considerable aumento de la diversidad del alumnado en las aulas.

Así, vemos que el profesorado ha de desempeñar sus funciones docentes y orientadoras para conseguir lo mejor de todos y cada uno de los estudiantes, cuando éstos, entre sí, son sumamente diferentes: alumnos interesados por su formación con expectativas de estudios superiores junto a otros desmotivados que viven su escolarización como una imposición legal más que como el ejercicio de un derecho; algunos, en riesgo de exclusión social que condiciona negativamente su vida escolar, personal y 
social; otros, con necesidades educativas especiales de muy distinta naturaleza (desde aquellas asociadas a diferentes formas de discapacidad hasta las derivadas de altas capacidades, pasando por toda una variada gama); así mismo, crece el número de adolescentes de origen inmigrante con necesidades educativas, también, muy diferentes entre sí. Esta breve enumeración de nítidas diferencias puede llevar al equívoco de entender diversidad asociada, únicamente, a sujetos especiales; algo propio de una concepción segregadora de la diversidad. Sin embargo, el concepto sobre el que se asienta el presente trabajo es propio de una interpretación inclusiva. Entendemos la diversidad, en sintonía con otros estudios (Fernández Enguita, 1990; Albericio, 1991; Parrilla, 1992 y 2002; Muñoz y Maruny, 1993; Garanto, 1994; Díaz Allué, 1999; García García, 1997, 2002 y 2005), como manifestación de la identidad de todo ser humano, como característica inherente a cada persona.

En consecuencia, el profesorado ha de atender a una población estudiantil sumamente heterogénea que requiere una educación de calidad. Y, justamente, conjugar calidad y equidad exige responder a esa diversidad de necesidades en el seno de una educación inclusiva. Esto significa, entre otras implicaciones, que la docencia en contextos educativos plurales representa mayor complejidad y dificultad que aquellos relativamente homogéneos: es posible que se agudicen problemas ya existentes, que los profesores tengan que enfrentarse a demandas desconocidas, que se cuestionen los fines de la educación a la luz de los recursos, apoyos y formación con los que cuentan. En definitiva, se ven abocados a una situación que les demanda una respuesta adaptada a las nuevas exigencias educativas. Esta realidad, y la considerable diversidad del alumnado a que da lugar, reclama de los docentes actitudes, competencias y funciones profesionales de naturaleza muy distinta a las inherentes a una educación secundaria no obligatoria como ha sido el tradicional Bachillerato. Esto unido a que el profesorado de Educación Secundaria no responde al mismo perfil profesional, ni pertenece a un único cuerpo docente, contribuye a complicar más, si cabe, lo relativo a esta etapa educativa.

Tras estas pinceladas que ponen de manifiesto la complejidad inherente a la diversidad del alumnado de la ESO y la necesaria respuesta educativa que exige, nos planteamos conocer, mediante un amplio estudio, cómo interpretan los profesores de la Comunidad Autónoma de La Rioja la problemática asociada a la diversidad. Este trabajo se enmarca en el contexto de una serie de investigaciones que, comenzando por estudiar la integración escolar a partir de su aplicación en España (Illán, 1989; Aguilera y otros, 1990; Parrilla, 1992) y teniendo en cuenta las realizadas en otros países (Booth, 1987; Corman y Gottlieb, 1987; Slavin y Madden, 1987), avanza por el camino -no exento de dificultades- de la educación inclusiva. Algunas de las aportaciones de mayor calado en este ámbito provienen de Australia, Estados Unidos y Reino Unido (Heshusius, 1984, 1986; Villa y Thousand, 1995; Booth y Ainscow, 1998, 2000). En España también se está produciendo una fecunda corriente bajo estos presupuestos; es el caso de estudios, entre otros, como los de Gairín, 2001; Durán y otros, 2002; ParriIla, 2002; Susinos, 2002; Echeita y Verdugo, 2004; Moriña 2004, Cardona, 2005; y García García, 2005. De modo más concreto, los trabajos que abordan la problemática suscitada por la diversidad en la ESO lo hacen desde variadas perspectivas y metodologías que contribuyen a desentrañar su complejidad y dificultad. Mencionaremos, 
únicamente, algunos como muestra de la riqueza que representa el tema. Para ello referiremos, en primer lugar, algunas de las investigaciones más significativas que estudian la diversidad en relación a la realidad de los Centros de Secundaria y, en segundo lugar, desde la perspectiva específica del profesorado de la ESO.

En el primer bloque destacamos los siguientes: Díaz Allué y otros (1997), en el marco de un amplio trabajo sobre Orientación en Educación Secundaria abordan, entre otros, el estudio de la función tutorial y sus posibilidades para atender a la diversidad, desde la óptica de los propios alumnos y profesores; Pujolás (1997, 1999) analiza el modelo de aprendizaje cooperativo como alternativa para desarroIlar una enseñanza que atienda las necesidades de todos los estudiantes; Díaz-Aguado y equipo (1999) se plantean el tratamiento dado a las diferencias culturales, la identificación de modelos educativos y pautas de actuación y su valoración; ParriIla y Gallego (1999) estudian un modelo de apoyo colaborativo entre profesores (GAEP) como alternativa a la interpretación del apoyo como práctica terapéutica profesional aislada; Álvarez Teruel (1999), desde la perspectiva de los profesores orientadores, averigua cómo llevan a cabo los IES la respuesta a la diversidad y, más concretamente, los Departamentos de Orientación; Álvarez Rojo y equipo (2002) tratan, entre otros objetivos, de conocer las actuaciones llevadas a cabo en relación a la diversidad en Centros de Secundaria, así como las opiniones y actitudes del profesorado al respecto; González Muñoz (2002) analiza el impacto en la realidad de las medidas legales emanadas de la LOGSE para responder a la diversidad; Pérez Gómez y Sola (2003), en una investigación acerca del desarrollo del currículo y factores de calidad en la ESO identifican, entre los principales problemas que condicionan su calidad, la dificultad de hacer compatible la comprensividad con la diversidad así como la desviación de vías de atención a la diversidad (por ejemplo, AC y PDC, Optativas) hacia prácticas discriminatorias con una tendencia a la homogeneización de los grupos.

En el segundo bloque, el más directamente relacionado con nuestra investigación porque se centra en el tema desde la perspectiva del profesorado, seleccionamos las siguientes: Forteza $(1998,1999)$ se plantea descubrir qué problemas tienen los profesores cuando deben atender a un alumnado heterogéneo y qué pueden hacer para afrontarlos; García Llamas (1999) estudia las necesidades formativas del profesorado y concluye, entre las más prevalentes, aquellas relativas a la atención a la diversidad como son las funciones de tutoría, la orientación académica y profesional del alumnado, la elaboración de proyectos educativos y curriculares centrados en el contexto, la evaluación y el trabajo en equipo; Arnaiz y otros (1999/2000) pretenden averiguar el cambio de actitudes hacia la diversidad como consecuencia de un programa de formación, concluyendo que, en términos generales, se produce un cambio positivo; Torres (2001) también analiza las necesidades de formación y propone una serie de estrategias para mejorar la formación permanente; De Prada (2002), en el marco de una investigación más amplia, estudia la opinión del profesorado acerca de las medidas más efectivas para atender la educación de todo el alumnado en el propio Centro: aborda la valoración de su sensibilidad y su posición ante la diversidad así como su opinión sobre la adecuación de medidas de atención a la diversidad de tipo organizativo, curricular, aca- 
démico y metodológico y sobre las que consideran más idóneas para su aplicación; el Instituto IDEA, Marchesi y Martín (2002), en el contexto de una amplia investigación sobre evaluación de la Educación Secundaria -desde la óptica de profesores, padres y alumnos-, analizan, entre otros varios temas, el contexto sociocultural, recursos disponibles, procesos desarrollados en cada Centro y aula, y valoraciones efectuadas por todos los sectores de la comunidad educativa entre las que destacan, como aspectos positivos, la buena valoración de la presencia de orientadores en los Centros y la posibilidad de ejercer la autonomía y, como negativos, el rechazo de la comprensividad en la ESO, el desánimo de los docentes, la falta de interés de los estudiantes y las considerables dificultades de los Centros de ambientes económica y socioculturalmente desfavorecidos.

Como se desprende de este somero análisis, la respuesta educativa a la diversidad en la ESO representa grandes desafíos para todas las instancias implicadas y suscita un considerable interés como ámbito de investigación. Por tanto, conscientes de ello, nos planteamos estudiar uno de los estamentos con mayor responsabilidad e incidencia en su aplicación práctica: el profesorado.

Este artículo refleja parte de una amplia y rigurosa investigación de la que se presenta una síntesis de aspectos imprescindibles tales como: una precisa identificación del objeto de estudio, los objetivos propuestos, las principales variables, la metodología seguida, el cuestionario como instrumento de trabajo y conclusiones derivadas del estudio. De este modo, se ofrece una visión de conjunto. Sin embargo, dada la riqueza de resultados obtenidos a éste seguirán otros varios en los que, de modo más detallado, se presentarán los resultados en los que se basan las conclusiones que aportamos. No obstante, el trabajo en su totalidad ya está disponible para su consulta (Jiménez Trens, 2003).

\section{Objeto de Estudio}

El propósito al que obedece este trabajo consiste en conocer y describir la problemática, experimentada por el profesorado de la Comunidad Autónoma de La Rioja que imparte la ESO, ante la diversidad del alumnado. Se trata de conocer con precisión qué piensan los docentes acerca de esa diversidad, cómo interpretan las posibilidades de respuesta educativa a la misma, qué dificultades y apoyos encuentran en el día a día de su trabajo, y cómo valoran su formación psicopedagógica para este cometido. Todo ello con la intención de que sean ellos mismos los que pongan de manifiesto sus pensamientos, deseos, valoraciones y propuestas al respecto. Cabe señalar, asímismo, que se pretende hacer una descripción de las opiniones del conjunto de los profesores y también detectar si existen diferencias significativas entre grupos de docentes según características de tipo personal (sexo y edad), profesional (colectivo docente y situación administrativa o laboral) y de centro (públicos y concertados). Para llevarlo a cabo hemos estructurado el contenido del trabajo en siete dimensiones: 1) Percepción de la comprensividad. 2) Percepción de la diversidad. 3) Valoración de las posibilidades de respuesta educativa. 4) Dificultades para responder a la diversidad. 5) Apoyo recibido para atender a la diversidad. 6) Formación psicopedagógica para responder a la diversidad y 7) Visión sintética de esta problemática. 


\section{OвJetivos}

En todos los objetivos destinados a recabar la opinión del profesorado, nos proponemos conocer la tendencia general así como detectar las diferencias entre grupos de profesores según características de tipo personal, académico, profesional y según el tipo de centro. Son los siguientes:

1. Describir las características de desarrollo de la docencia en relación a la diversidad, en el propio centro: perfil del alumnado, departamento de Orientación y programas específicos de atención a la diversidad.

2. Identificar su opinión sobre la comprensividad en la ESO acerca de los aspectos siguientes: a) consecuencias de la comprensividad para los estudiantes; y b) conveniencia del inicio de itinerarios: curso y edad.

3. Conocer cómo perciben la diversidad concretada en los aspectos siguientes: a) estudiantes a los que se refiere la diversidad; b) finalidades de la atención a la diversidad; c) responsables de la atención a la diversidad; d) sentimientos, creencias o estados de ánimo ante esta realidad; e) opinión general sobre los alumnos con discapacidad.

4. Analizar la valoración de las posibilidades de respuesta educativa a la diversidad: 1 ) apreciación global de una serie de medidas; 2) valoración de medidas comunes (documentos de planificación de centro y de aula; criterios tenidos en cuenta en la programación y desarrollo de las clases; la práctica de la evaluación como medida común; materias optativas, como otra forma de atender a la diversidad); 3) autopercepción para responder a la diversidad; 4) opiniones sobre las medidas de carácter específico (Adaptaciones Curriculares Individuales y Programas de Diversificación Curricular).

5. Identificar las principales dificultades para atender a la diversidad, tanto las atribuidas al contexto y de carácter organizativo, como las experimentadas ante determinados perfiles o grupos de alumnos.

6. Detectar la valoración de los apoyos recibidos para afrontar esta problemática: a) de órganos personales e institucionales; y b) del Departamento de Orientación.

7. Conocer cómo valoran su formación psicopedagógica y qué necesidades de formación experimentan para adaptar la enseñanza a las nuevas exigencias educativas.

8. Identificar la percepción sintética sobre esta temática.

9. Elaborar un instrumento para conocer la percepción de estas cuestiones.

\section{IDENTIFICACIÓN DE LAS VARIABLES}

Las variables de este estudio se clasifican en dos bloques. El primero corresponde a las de identificación o clasificatorias que se refieren a las características de los sujetos estudiados. El segundo refleja la percepción del profesorado, en coherencia con las dimensiones y objetivos enunciados. Como el tema es amplio, complejo y polémico, se han confeccionado un número muy amplio de variables que representan muy diversos argumentos correspondientes a muy variadas opiniones. Así 
mismo, se han determinado los valores que pueden adoptar mediante escalas valorativas, tipo Likert, con una gradación de uno a cinco. A continuación, mencionaremos únicamente las principales de cada dimensión (para una información más completa, véase Jiménez Trens, 2003: 223-247).

Para identificar su percepción acerca de la comprensividad en la ESO se han analizado las opiniones que tienen sobre las consecuencias de la enseñanza comprensiva para los estudiantes y sobre cuándo consideran conveniente iniciar itinerarios diferentes. Para ello, en primer lugar, se han propuesto reflexiones en dos sentidos contrapuestos que recogen opiniones positivas y negativas tales como: garantía de igualdad de oportunidades para todos los jóvenes; resultan perjudicados los más capaces, etc. y, en segundo, se ofrecen todas las posibilidades de comienzo de itinerarios desde primer curso a cuarto y en las diferentes edades.

Para conocer su percepción acerca de la diversidad, tema muy amplio sobre el que se pueden mantener ideas bien distintas y con implicaciones prácticas muy diferentes, se han tomado como referencia tres modelos de interpretación: selectivo o segregador, compensador de las desigualdades, e inclusivo o promotor de educación integral para todos. Para analizar las correspondientes variables, se han estructurado en dos partes. En la primera, de acuerdo a los tres modelos indicados, se estudia qué concepto tienen de la diversidad en general, focalizándolo en: a) los estudiantes a los que consideran "diversos"; b) finalidad de la atención a la diversidad; c) responsables de la misma; y d) sentimientos, creencias o estados de ánimo ante la diversidad. En la segunda, se recogen tendencias de opinión positiva y negativa acerca de los alumnos con discapacidad integrados en los centros de secundaria.

El análisis de su valoración de las posibilidades de respuesta educativa, se ha efectuado, básicamente, de dos modos. En primer lugar, se ha tratado de que puedan manifestar qué apreciación global les merece una amplia serie de posibilidades existentes en la dinámica de los centros y que incluyen medidas de muy variado carácter (curricular, organizativo, de orientación, didáctico, etc.). En segundo lugar, se ha profundizado en las medidas comunes $u$ ordinarias y en las extraordinarias. Respecto al análisis de las medidas ordinarias, se ha tratado de identificar: a) cómo valoran la importancia de los documentos de planificación de centro y aula (Proyecto Educativo, Proyecto Curricular, Plan de Orientación Académica y Profesional, etc.); b) cómo consideran su propia práctica para responder a la diversidad cuando hacen sus programaciones de clase y en su práctica docente habitual; c) cómo consideran su propia práctica de la evaluación (si les sirve para individualizar la enseñanza, etc.); d) la opinión acerca de las materias optativas (si las que se ofertan responden a los intereses y necesidades del alumnado, o si, por el contrario, 'se quita' tiempo que sería más útil dedicar a materias más importantes, etc.); e) autopercepción de la capacidad de afrontar la diversidad (sólo me adapto al alumnado tipo medio; oprocuro atender no sólo al promedio sino también niveles muy dispares; etc.). Respecto a las medidas extraordinarias, se estudian las opiniones sobre las Adaptaciones Curriculares Individuales y sobre los Programas de Diversificación Curricular.

La identificación de las dificultades se ha estructurado en torno a dos tipos de naturaleza diferente. Uno de ellos se refiere a las variables de tipo contextual o de carácter organizativo, tales como excesivo número de alumnos por aula; grupos- 
aula con muchos alumnos problemáticos; escasez de recursos didácticos; horarios rígidos que impiden la organización de alguna de las medidas; demasiados problemas de disciplina; ausencia de coordinación del trabajo docente para organizar la atención la diversidad; etc. El otro se refiere a la percepción que los profesores tienen de sus dificultades con relación a ciertos perfiles de alumnos tales como con graves problemas de conducta; pertenecientes a minorías étnicas; con deficiencias; con altas capacidades o superdotados; etc.

El apoyo recibido para atender a la diversidad se ha analizado desde dos ópticas complementarias. Por un lado, se trata de variables que indican la valoración global de apoyos que pueden provenir de variadas fuentes; algunas, de carácter personal, otras, institucional, tanto internas como externas al propio centro (algún compañero,tutores; equipo docente; profesores de Apoyo; departamento de Orientación; etc. Por otro, dada la relevancia de la orientación para la atención a la diversidad, hemos recabado su opinión acerca de la importancia que atribuyen a este departamento bien a través de la ayuda al profesorado para este cometido, o bien en relación al alumnado que lo necesite (en la mejora de procesos de enseñanza/aprendizaje de todos los alumnos; para la prevención, detección y tratamiento de problemas del alumnado, etc.)

Para conocer cómo valoran su formación psicopedagógica se han definido variables que permiten apreciar su grado de calidad (de mala a muy buena) en distintos momentos clave (durante la carrera, permanente, etc.). Para averiguar qué necesidades de formación tienen se presentan variables nominales para que elijan todas las que estimen conveniente; algunas de estas propuestas son las siguientes: estrategias de enseñanza/aprendizaje para adaptar la enseñanza común a las diferencias individuales; Adaptaciones Curriculares para superdotados y para los de graves dificultades de aprendizaje.

Para identificar qué visión sintética tienen sobre esta problemática se han definido dos grupos de variables. En uno se someten a la consideración de los profesores varias propuestas que sintetizan aspectos relevantes relativos a la Administración, al profesorado, al ambiente de centro y a la percepción general de la problemática como soluble o insoluble. En el otro, se ofrece la posibilidad de que manifiesten libremente cualquier sugerencia, enfoque y alternativas de solución en relación al tema.

\section{Metodología}

\section{Población y muestra}

La población está constituida por el conjunto de profesoras y profesores que imparten Educación Secundaria Obligatoria en toda la geografía riojana. Ha sido estimada en 1.257 profesores. Pertenecen a tres colectivos: Profesores de Educación Secundaria, Maestros de Educación Primaria adscritos a la ESO y Profesorado Técnico de Formación Profesional; tienen distinta situación administrativa o laboral: funcionarios con destino definitivo o provisional, interinos, etc.; son profesores de la red pública y de la privada. Dadas las características de la población: finita, de reducido volumen y asequible localización geográfica, se decidió acceder a todo el profesorado. No obstante, la muestra productora de datos, finalmente, ha sido de 432 
profesores, lo que representa el 34,4\% de la población. Muestra considerada suficiente, incluso superior a la que algunos expertos señalan como habitual en este tipo de estudios. Aunque se trata de una muestra incidental, existe representación de todos los centros y de todos los grupos de profesores.

Del total de la muestra, el 54,4\% son hombres y el 45,1\% mujeres; una mayoría $(54,6 \%)$ tiene entre 35 y 50 años. Así mismo, un 65,5\% son profesores de Educación Secundaria, frente a un $27,3 \%$ que son maestros adscritos a la ESO; el 53,2\% son funcionarios definitivos. Y en cuanto a la titularidad de los centros, un $69 \%$ trabaja en IES frente a un 30,6\% que lo hace en centro concertado.

\section{Diseño de investigación}

Este diseño se realiza mediante el método de encuesta y el cuestionario como instrumento de trabajo. Dado el carácter descriptivo de esta investigación consideramos que la encuesta es un método científico apropiado porque es una vía oportuna para acceder al pensamiento de los profesores y se adecúa a las características del objeto de estudio. Así mismo, proporciona un buen nivel de rigor y calidad y podemos afrontar las condiciones de realización práctica (tiempo, recursos, etc.). Permite obtener información relevante, general y matizada, acerca del problema enunciado, sin ejercer ninguna manipulación sobre las variables del mismo.Se puede considerar que es un estudio de tipo ex post facto, ya que en nuestra mano no está manipular las variables independientes para descubrir qué efectos produce en el estado de opinión del profesorado, ni tampoco podemos asignar los profesores, de modo aleatorio, a grupos controlados.

Únicamente se trata de descifrar y constatar sus reflexiones acerca de la diversidad y de su posible respuesta y, en todo caso, averiguar si existen relaciones entre éstas y su pertenencia a alguno de los grupos analizados. En resumen, una vez obtenidos los datos, en primer lugar, se pretende un estudio descriptivo general de todas las respuestas con un análisis de tendencias. Seguidamente, se utilizan diferentes grupos de contraste; los principales son: los grupos de profesores de centros públicos y concertados, los de Secundaria, Primaria adscritos a la ESO y Formación Profesional, los de distinta edad, de diferente situación administrativa o laboral, etc.

\section{El Cuestionario como instrumento de trabajo}

Revisada la bibliografía existente y habiendo comprobado que no había ningún instrumento que respondiera a nuestros objetivos, hemos procedido a elaborar uno propio: un cuestionario. Éste nos permite analizar todas las dimensiones e indicadores identificados en el estudio, abarca a todos los grupos de profesores y es viable. Consta de 44 preguntas de las que, las 21 primeras, son relativas a la identificación de las características de los encuestados y, las 23 siguientes, se refieren a las dimensiones e indicadores del estudio. En su mayoría, son cerradas, intercaladas con alguna de tipo abierto. Se ha decidido un modelo de medición tipo Likert. En cuanto al número de respuestas por ítem, se ha optado por cinco (dos valoraciones en sentido positivo, dos en sentido negativo y una respuesta central que indica indecisión). Por último, tras someter el instrumento a los necesarios controles de validez de conteni- 
do y tras los análisis efectuados de validez de constructo y de fiabilidad, se concluye que el instrumento reúne los niveles de validez y fiabilidad exigidos para una correcta interpretación de los datos.

\section{Conclusiones}

A continuación presentamos una síntesis de las conclusiones derivadas del estudio.

\section{Percepción de la comprensividad}

- Los resultados nos indican que perciben la comprensividad como un tema polémico, aunque se decantan por una visión más bien negativa. Aceptan, en general, los posibles perjuicios académicos y reconocen relativamente -con opiniones divididas- los potenciales valores socioeducativos que representa. Así, una amplia mayoría (casi el 77\%) considera el riesgo del descenso del nivel académico del conjunto de los estudiantes; sin embargo, al interpretar los posibles valores sociales, se observa una clara disparidad de opiniones ya que, casi un 43\%, afirma que es conveniente para que aprendan a convivir en democracia, pero casi un 36\% cree que no; opinión, por tanto, dividida con tendencias marcadas hacia los extremos, que indica lo controvertido del tema y que, probablemente, tenga un correlato con el clima social existente. Por tanto, la causa de este rechazo parece estar en razones de tipo académico polarizadas en el conjunto de los estudiantes y en los más capaces, que no consideran compensadas por los beneficios de tipo socioeducativo. De acuerdo a esta conclusión sería interesante comprobar, en futuros estudios, si ese rechazo sería menor en el caso de que se asegurara una formación académica de calidad para todos y cada uno de los estudiantes.

- Mediante los estudios diferenciales realizados, en general, no se detectan apenas diferencias y, cuando se observan, aunque estadísticamente significativas, tampoco son de gran magnitud ni ofrecen una relevancia especial. No obstante, merece destacarse que los profesores de Educación Secundaria manifiestan de modo más acentuado la opinión negativa, en contraste con el grupo de maestros adscritos a la ESO. La razón de ello podría encontrarse en la diferente 'cultura profesional' a la que pertenecen, en la formación recibida y, sobre todo, en las distintas expectativas de desarrollo profesional generadas en la carrera de magisterio o en la correspondiente licenciatura. No olvidemos el escaso peso que tiene la formación psicopedagógica en los profesores de Secundaria y recordemos que sus estudios se dirigen principalmente a formar especialistas en el propio ámbito de conocimiento que les capaciten para 'crear' ciencia, mucho más que para enseñar a aprenderla y amarla a unos adolescentes -en gran número desinteresados-. Esta tendencia de opinión se ve confirmada en otros trabajos (Marchesi y Monguilot, 2001; IDEA, Marchesi y Martín, 2002; De Prada, 2002).

- Esta postura controvertida ante la comprensividad se confirma cuando se manifiestan acerca de cuándo consideran conveniente que los alumnos inicien itinerarios diferentes. Llama la atención la gran variabilidad de la respuesta, ya 
que no hay una clara mayoría que se decante por un curso y edad. Lo que sí se observa es una tendencia superior a las demás en marcarlo en $3^{\circ}(39,4 \%)$ y a la edad de 14 años (34,5\%); y si sumamos los partidarios de iniciar en $3^{\text {o }}$ y 4º , obtenemos más de un $61 \%$ que sí lo harían. También es conveniente resaltar que no son partidarios de comenzar tempranamente (en $1^{\circ}$ ó $2^{\circ}$ ) esa diferenciación. Respecto a estos datos, cabe concluir que apuntan en la misma dirección, aunque de manera menos acusada, que los obtenidos en otros estudios (INCE, I, 1998:150; Marchesi y Monguilot, 2001: 70).

\section{Percepción de la diversidad}

- Respecto a la diversidad, hemos sabido cómo la perciben los profesores de la muestra, en relación a los modelos estudiados (selectivo, compensador e inclusivo). Nuestros resultados indican una tendencia de opinión sumamente interesante. Previamente, recordemos que son muchos los profesores que perciben una considerable diversidad en el alumnado de sus centros. Este hecho da más valor, si cabe, a sus opiniones, por la orientación hacia un modelo inclusivo que manifiestan.

- En cuanto al concepto de diversidad, la coherencia en los resultados de las distintas variables es muy interesante y prueba de sinceridad en las respuestas. A diferencia de otros estudios (INCE, I, 1998; IDEA, Marchesi y Martín, 2002), en los que parece que los profesores no lo tienen excesivamente claro, en nuestro trabajo sí se revela claro este concepto y, más aún, apunta hacia una percepción inclusiva de la diversidad. Veamos, de modo más concreto, estos resultados.

- Así, respecto a qué estudiantes son 'diversos', su opinión se decanta por considerar que, de modo general, son todos y, de modo específico, algunos. Cuando se manifiestan sobre qué finalidades tiene la atención a la diversidad, lo hacen, en una gran mayoría, reconociendo que consiste en potenciar el desarrollo máximo de las capacidades de todos y cada uno de los alumnos. Cuando afirman quiénes deben ser los responsables, contundentemente dicen que todos (profesores, tutores, departamento de orientación, profesores especialistas en Pedagogía Terapéutica o Logopedia) trabajando en equipo, cada uno desde su ámbito de actuación. Nos parece un indicador interesante de la madurez que demuestran nuestros profesores. Podían haber dicho que era competencia sólo de los especialistas o del Departamento de Orientación. Pero no; asumen su responsabilidad, reconociendo la necesidad de que se impliquen todos.

- En cuanto al ámbito de la afectividad (sentimientos, creencias o estados de ánimo), manifiestan una respuesta paradójica entre los sentimientos positivos y estados satisfactorios junto a un lamento generalizado manifestado, sobre todo, mediante la expresión libre. Probablemente, están evidenciando la distancia entre los deseos (relativos a lo positivo) y la realidad (sentimientos de impotencia, frustración, angustia, etc.). Como contraste entre deseo y realidad, se puede observar la masiva afirmación de que les gusta que los jóvenes, en un medio plural, aprendan a cooperar, así como a dar y pedir ayuda; sin embargo cuando deben decidir que, aunque sea más duro trabajar con alumnado heterogéneo, merece la pena el esfuerzo, poco más del 40\% afirma estar dispues- 
to. Pensamos que la realidad de los centros y su propio compromiso en este empeño, incide sobre el estado de opinión vinculado a aspectos de la vida afectiva.

- La opinión que expresan sobre los alumnos con discapacidad integrados en los centros de Secundaria, parece indicar un relativo desconocimiento de los mismos, ya que la mitad de la muestra engrosa el grupo de los indecisos o no contestan. Sin embargo, nos parece muy esperanzador ese casi 50\% que reconoce que la convivencia con estos alumnos/as 'humaniza' a los demás estudiantes.

- En cuanto a los estudios diferenciales, los grupos de profesores que manifiestan una percepción ligeramente más integradora/inclusiva de la diversidad, son los menores de 35 años, en contraste con los mayores de 50; los interinos, en contraste con los funcionarios; y los maestros en contraste con los profesores de Educación Secundaria.

\section{Valoración de las posibilidades de respuesta educativa}

- Las medidas organizativas son las mejor valoradas por los profesores de nuestra muestra. Concretamente, las mejor consideradas son las siguientes: los desdoblamientos de grupo en algunas áreas para refuerzo de quién lo necesite y la organización del trabajo docente en equipo para responder mejor a la diversidad (más del 75\% les atribuyen bastante o mucha importancia). Nuestros resultados coinciden con los del trabajo realizado por De Prada (2002: 154). También ella encuentra que las medidas organizativas -y de ellas, el establecimiento de grupos de apoyo para las materias instrumentales-, son las mejor consideradas por los profesores de su muestra. Sin embargo hay una diferencia muy importante entre sus resultados y los nuestros. $Y$ es que la segunda medida mejor considerada por sus profesores es el establecimiento de grupos homogéneos por capacidades. En nuestro estudio, valoran la necesidad del concurso de todos, que puede adquirir formas organizativas distintas, pero que no reclaman la segregación del alumnado en grupos homogéneos.

- Le siguen en la valoración algunas medidas curriculares de carácter específico: Programas de Garantía Social, Programas de Diversificación Curricular y Adaptaciones Curriculares Individualizadas que, como se ve, también implican cambios organizativos específicos.

- Así mismo resulta muy bien valorada la práctica de la orientación individualizada para todo el alumnado. Resultados coherentes con el reconocimiento de la función de los departamentos de orientación, como se verá más adelante, que resulta muy bien considerado.

- De modo coherente con los resultados obtenidos sobre el concepto de atención a la diversidad, observamos una tendencia a considerar mejor las fórmulas que no requieren una fuerte segregación para responder a la diversidad, ya que entre las medidas peor valoradas se encuentran la repetición de curso junto a los grupos homogéneos.

- Si profundizamos en el análisis de las medidas comunes, vemos que destacan las Programaciones de Aula como una de las mejor consideradas. Así mismo 
la valoración en su propia práctica de una serie de criterios que permiten la atención a la diversidad, desde la realización de su trabajo habitual, mediante la programación y el desarrollo cotidiano de las clases; entre los que más frecuentemente identifican (un 80 \% o más), están los siguientes: que las actividades de enseñanza-aprendizaje sean variadas, que las clases resulten apropiadas para todos y el intento de fomentar el estudio independiente o trabajo personal. Resultados coherentes con lo que venimos afirmando acerca de una tendencia de opinión orientada más a la inclusión que a la segregación.

- También atribuyen a la evaluación unas funciones que facilitan la respuesta a la diversidad: Sirve, en su opinión, para valorar el progreso individual, para conocer sus posibilidades y para preparar actividades de refuerzo y ampliación. Funciones que se reconocen más en la línea de evaluar para conocer, comprender y ayudar, que para seleccionar y clasificar.

- Cuando se trata de averiguar cómo se perciben a sí mismos en relación a la diversidad y el contexto en el que desarrollan su trabajo, es decir, se les pregunta por su propia capacidad para atender la diversidad, en la realidad de su trabajo, observamos también una muy interesante respuesta: se reconocen con capacidad, pero con dos condiciones: el apoyo de la Administración y el trabajo coordinado con todos los profesionales implicados en la educación. En relación a sus intenciones, afirman que procuran atender no sólo al promedio sino también a los de niveles muy dispares.

- EN RESUMEN: se aprecia una tendencia al reconocimiento y la apreciación de todas las posibilidades que ofrecen las medidas comunes o de carácter preventivo de atención a la diversidad. Percepción congruente con la interpretación de la diversidad, expuesta anteriormente. Estos resultados nos indican un pensamiento reflexivo por parte del profesorado orientado hacia la inclusión, en cuanto a que se plantea el tema de la diversidad aprovechando todas las oportunidades de atenderla, sin necesidad de recurrir como primera, y quizás única solución, a las medidas de carácter específico o extraordinario. Antes, al contrario, están pensando en agotar primero estas posibilidades. Por grupos, los profesores que parecen identificarse en mayor medida con estas posiciones son los menores de 35 años frente a los mayores de 50 años; los interinos frente a los funcionarios; los maestros frente a los profesores de Educación Secundaria y los de centros concertados frente a los públicos.

- Cuando se analiza cómo perciben las medidas específicas observamos las siguientes tendencias:

a) Las Adaptaciones Curriculares Individualizadas (ACls) son, globalmente, muy bien valoradas; sin embargo, se muestran muy críticos a la hora de referirse a las condiciones organizativas y oportunidades para su aplicación práctica. Una vez más ponen de manifiesto la discordancia entre una valoración, más bien de carácter teórico, y las posibilidades que reconocen de llevarlas a la práctica en su contexto habitual de trabajo.

b) Los Programas de Diversificación Curricular (PDC), también resultan muy bien valorados en cuanto a la importancia que les atribuyen, y porque consideran que favorecen la atención individualizada y que son muy útiles para 
atender la diversidad. Sin embargo, piensan que esperar a los 16 años para cursarlos es demasiado tarde.

\section{Dificultades para responder a la diversidad}

- Las dificultades que, de modo más generalizado, manifiestan los profesores, representan problemas cuya solución no depende de ellos; se trata de dificultades contextuales, consecuencia de las decisiones de otros (Administración y familias, sobre todo). Concretamente, las que exponen con mayor frecuencia (pasando del 80\% y del 70\%) son las siguientes: Grupos-aula con muchos alumnos problemáticos, excesivo número de alumnos por aula, ausencia de colaboración por parte de las familias y falta de apoyo por parte de la Administración. Aquellos problemas que requieren, para su solución, la implicación del centro y de los propios profesores, son reconocidos por un número, aunque elevado, sustancialmente menor que en los casos anteriores: es el caso de la ausencia de un ambiente o clima de centro favorable a la respuesta educativa adaptada a las diferencias.

Parece como si cargaran más las tintas en la atribución de dificultades o problemas a instancias exteriores a ellos mismos, de los que, en consecuencia, no se sienten responsables. La respuesta libre ratifica sobradamente estos resultados.

- Las dificultades que manifiestan en relación a ciertos perfiles de alumnos se polarizan, como era de esperar, en torno a los que presentan graves problemas de conducta y los que están en clase por obligación (a más del 70\% de profesores les ocasiona bastante o mucha dificultad). En esta especie de ranking de dificultades, siguen a considerable distancia los que desconocen nuestro idioma $(47,3 \%)$ y los que tienen deficiencia mental $(45,2 \%)$.

- Analizando las respuestas por grupos de profesores, destacan los de centros públicos que tienen una percepción más generalizada de las dificultades. Los problemas señalados más frecuentemente son los siguentes: ausencia de un ambiente favorable a la diversidad, demasiados problemas de disciplina, grupos-aula con muchos alumnos problemáticos, ausencia de coordinación del trabajo docente y muchos grupos a los que impartir docencia. Estos profesores, también manifiestan más dificultades que sus colegas de la privada, en relación a ciertos perfiles de alumnos: que desconocen idioma, con deficiencia visual, etc.

\section{Apoyo recibido para atender a la diversidad}

- La valoración que efectúan de los apoyos recibidos en sus responsabilidades de atender a la diversidad es más bien escasa. El que más reconocen es el que procede de algún compañero de modo más o menos informal. (46,6\%); valoración parecida les merece el de los tutores y el del Departamento de Orientación. Este último es el mejor valorado de todos los órganos de carácter institucional. Así mismo, consideramos que constituye un motivo de reflexión el escasísimo valor concedido a la Administración y la Inspección, así como a la Universidad (4,2\% y $1,4 \%$, respectivamente). 
- Cuando se considera la importancia del departamento de orientación, en el campo de la atención a la diversidad, resulta muy bien valorado. No obstante, los profesores destacan las competencias más vinculadas con las necesidades educativas especiales: evaluación psicopedagógica...; prevención, detección y tratamiento de problemas; organización de la respuesta a la diversidad, que con el asesoramiento de los profesores para la mejora de la enseñanza y el aprendizaje de la totalidad del alumnado. Parece que siguen considerando a este departamento más relacionado con aspectos específicos de atención a la diversidad que con los generales.

\section{Formación psicopedagógica para responder a la diversidad}

- Un elevado porcentaje de profesores $(65,3 \%)$ considera nula o mala la formación recibida durante la carrera. Se observa la tendencia a valorar algo mejor la formación permanente que la inicial; así mismo, la valoración de los Maestros, aún siendo mala, es ligeramente mejor que la de los Profesores de FP y la de los Profesores de Educación Secundaria. También se confirman estos datos en la visión sintética final, cuando un $57,8 \%$ de profesores no se reconoce bien preparado para atender a la diversidad.

- En cuanto a las necesidades que manifiestan, en torno al 60 \% o más reclaman formación en: estrategias de enseñanza-aprendizaje para adaptar la enseñanza común a las diferencas individuales y Adaptaciones Curriculares para superdotados y para los que tienen graves dificultades. El resto de necesidades, también son ampliamente manifestadas, ya que entre un 50 y un $40 \%$ de profesores las exponen.

Nos parece muy interesante (y concordante con los datos anteriores) la demanda, en primer lugar, de una formación para ofrecer una respuesta individualizada desde el marco educativo común. Indica, en nuestra opinión, su posición más cercana a una postura de inclusión que a otra de exclusión. Y ello tampoco es obstáculo para que sean conscientes de las necesidades específicas y tratamiento extraordinario que requieren los superdotados y los de graves dificultades. Mediante la respuesta libre piden, sobre todo, formación para saber tratar a los alumnos conflictivos y a los carentes de todo interés por estudiar; en coherencia con estas inquietudes, también reclaman formación psicológica para preservar su equilibrio y salud mental.

\section{Visión sintética sobre esta problemática}

Cabe mencionar, como resultado más destacado, aunque no inesperado, la insuficiente dotación de medios y recursos, así como la inadecuación de la normativa de la Administración.

Sin embargo, nos parece muy esperanzador que, a pesar de haber dibujado un panorama bastante sombrío (en la formación, en las dificultades, en la falta de apoyo, etc.), haya un 40,3\% de profesores que reconocen que el profesorado, en general, tiene buena disposición para afrontar los problemas que acarrea la diversidad. Resultado que relacionamos, por su coherencia, con ese 44,2\% que afirma que, en general, los problemas originados por la diversidad tienen solución. Sería 
interesante descubrir qué ha llevado a esos profesores a opinar de ese modo. Y más, todavía, ampliar esa cantidad y lograr, poco a poco, avances en la respuesta educativa de calidad para todos y cada uno.

\section{BibLiOgRAFíA}

AGUILERA, M. J.; ÁlVAREZ, K.; BABIO, M.; COLL, C.; ECHEITA, G.; GALÁN, M.; MARCHESI, A.; MARTIN, E. y MARTÍNEZ-ARIAS, R. (1990). Evaluación del programa de integración escolar de alumnos con deficiencias. Madrid: CIDE / MEC. ALBERICIO, J. J. (1991). Educar en la diversidad. Madrid: Bruño.

ÁlVAREZ ROJO, V., RODRÍGUEZ, A., GARCíA, E., GIL. J., LÓPEZ, I., ROMERO, S. PADILLA, M. T., GARCÍA, J. y CORREA, J. (2002). La atención a la diversidad en los centros de enseñanza secundaria: estudio descriptivo en la provincia de SeviIla. Revista de Investigación Educativa, 20 (1), 225-245.

ÁLVAREZ TERUEL, J. D. (1999). La atención a la diversidad en la ESO. Papel del Departamento de Orientación y el Orientador Escolar. Análisis de la situación en la provincia de Alicante. Tesis Doctoral. Murcia: Universidad de Murcia.

ARNÁIZ, P., CASTEJÓN, J. L., GARRIDO, C. F. y ROJO, A. (1999-2000). Evaluación del cambio de actitud del profesorado tras su participación en un programa de atención a la diversidad. Revista Enseñanza. Anuario Interuniversitario de Didáctica, 17-18, 309-324.

BOOTH, T. (1987). Integrating Special Education. En BOOTH, T. y POTTS, P. (eds.). Integrating Special Education. Oxford: Basil Blackwell.

BOOTH, T. y AINSCOW, M. (eds.) (1998). From them to us: an international study of inclusion in education. London: Routledge.

- (2000). The index for inclusion: developing learning and participation in schools. Bristol: Centre for Studies on Inclusive Education (CSIE).

- (2002). Guía para la evaluación y mejora de la Educación Inclusiva. DesarroIlando el aprendizaje y la participación en las escuelas. Madrid: Consorcio Universitario para la Educación Inclusiva.

CANGA, A. I. y PASCUAL, M. A. (1998). Resultados en torno a la implementación de los Programas de Diversificación Curricular en la Educación Secundaria. En PÉREZ PÉREZ, R. (coord.), PASCUAL SEVILLANO, A. y ÁLVAREZ GARCíA, M. C. Educación y Diversidad. XV Jornadas Nacionales de Universidad y Educación Especial. Vol II. Oviedo: Universidad de Oviedo. Departamento de Ciencias de la Educación, 687-705.

CARDONA, M. C. (2005). Creencias, percepciones y actitudes hacia la inclusión: una síntesis de la literatura de investigación. En JIMÉNEZ, C. (coord.). Pedagogía Diferencial. Diversidad y equidad. Madrid: Pearson educación, 239-266.

CORMAN, L. y GOTTLIEB, J. (1987). La integración de niños mentalmente retrasados: Una revisión de la investigación. Revista de Educación, 1, 75-102.

DE PRADA, M. D. (2002). La Atención a la Diversidad en la Educación Secundaria Obligatoria. Marco organizativo, curricular y metodológico: estudio comparativo a nivel europeo, autonómico y local. Madrid: MECD. 
Diagnóstico del Sistema Educativo. 1997. 1, Elementos para un diagnóstico del Sistema Educativo Español: Informe global Madrid: Ministerio de Educación y Cultura, Instituto Nacional de Calidad y Evaluación, 1998. (Estudios e informes).

DÍAZ-AGUADO, M. J. (dir.); GIL, J. A.; JIMÉNEZ, R.; SACRISTÁN, A. y cols. (1999). Diversidad cultural e igualdad escolar. Un modelo para el diagnóstico y desarrollo de actuaciones educativas en contextos escolares multiculturales. En CIDE. Premios Nacionales de Investigación Educativa 1998. Madrid: CIDE, 15-32.

DÍAZ ALLUÉ, M. T. (1999). Orientación educativa y atención a la diversidad del alumnado de Educación Secundaria Obligatoria. Bordón, 51, (1), 115-124

DÍAZ ALLUÉ, M. T., CARBALLO, R., FERNÁNDEZ DÍAZ, M. J. y GARCÍA NIETO, N. (1997). Orientación en Educación Secundaria. Situación actual y prospectiva. Revista Investigación Educativa, 15 (2), 9-83

DURÁN, D. y otros (2002). "Index for Inclusion". Una guía para la evaluación y mejora de la Educación Inclusiva. Contextos Educativos, vol. 5.

ECHEITA, G. y VERDUGO, M. A. (coords.) (2004). La Declaración de Salamanca sobre Necesidades Educativas Especiales 10 años después. Valoración y prospectiva. Salamanca: INICO.

FERNÁNDEZ ENGUITA, M. (1990). Juntos pero no revueltos. Ensayos en torno a la reforma de la educación. Madrid: Visor.

FORTEZA, M. D. (1998). L'atenció a la diversitat segon cicle de l'educació secundària obligatòria. La distància entre la realitat i el desig. Un estudi de cas. Tesis Doctoral. Departamento de Ciencias de la Educación de la Universitat de les Illes Balears.

- (1999). Controversias e interrogantes en torno a la diversidad en Secundaria. Las voces de los implicados. Revista de Educación Especial, 26, 7-42.

GAIRÍN, J. (2001). Una escuela para todos: Un reto social y educativo. En SIPÁN COMPAÑÉ, A. (coord.). Educar para la diversidad en el siglo XXI. Zaragoza: Mira Editores, 241-266.

GARANTO, J. (1994). Concepto de la diversidad e implicaciones psicopedagógicas. Jornades sobre Orientació Psicopedagògica i atenció a la diversitat (5, 1994, Barcelona). Barcelona. Associació Catalana d'Orientació Escolar i Profesional, 17-28.

GARCÍA GARCÍA, M. (1997). Educación Adaptativa. Revista de Investigación Educativa., 15 (2), 247-271.

- (2002). Atención a la diversidad en Educación Secundaria Obligatoria. EduPsikhé: Revista de Psicología y Psicopedagogía, 1 (2), pp 225-248

- (2005). Educación Adaptativa y escuela inclusiva: una forma de atender las diferencias de todos los esudiantes. En C. JIMÉNEZ (Coord.). Pedagogía Diferencial. Diversidad y equidad. Madrid: Pearson educación, pp 3-31

GARCÍA LLAMAS, J. L. (1999). Análisis y valoración de necesidades formativas del profesorado. En Premios nacionales 1998 a la innovación educativa 1998. Madrid: CIDE, 211-234.

GONZÁLEZ MUÑOZ, M. C. (2002). Comprensividad, diversidad y calidad en las aulas. En Informe Educativo 2002. La calidad del sistema educativo. Madrid: Fundación Hogar del Empleado, Santillana, 170-188.

HESHUSIUS, L. (1984). Why would they and I want to do it? A Phenomenologicaltheoretical view of special education. Learning Disabilities Quarterly, 7, 363-368. 
- (1986). Paradigms Shifts and Special Education: A response to Ulman and Rosenberg. Exceptional Children, 461-465.

IDEA, MARCHESI, A. y MARTÍN, E. (comp.) (2002). Evaluación de la educación secundaria. Fotografía de una etapa polémica. Madrid: Fundación Santa María, SM.

ILLÁN, N. (1989). Los profesores y la integración escolar. Valencia: Nau Llibres.

INCE (1998). Elementos para un diagnóstico del Sistema Educativo Español. Informe global. Vol. 1. Diagnóstico del Sistema Educativo. La escuela secundaria obligatoria, 1997. Madrid: MEC/INCE.

JIMÉNEZ TRENS, M. A. (2003). El profesorado de la Educación Secundaria ante la diversidad del alumnado en la etapa obligatoria. Tesis Doctoral. Madrid: Universidad Complutense de Madrid. Disponible en: http://www.ucm.es/BUCM/tesis/edu/ ucm-t26875.pdf [última consulta: 22/03/2006].

MARCHESI, A. y MONGUILOT, I. (2001). La opinión de los profesores sobre la calidad de la educación. Madrid: Fundación del Hogar del Empleado.

MORIÑA, A. (2004). Diversidad en la escuela: diseño, desarrollo y valoración de una propuesta de formación para el profesorado. Organización y Gestión Educativa, 2, 32-34.

MUÑOZ, E. y MARUNY, L. (1993). Respuestas escolares. Cuadernos de Pedagogía, 212, pp 11-14.

PARRILLA LATAS, A. (1992). La integración escolar como experiencia institucional. Sevilla: Universidad de Sevilla, Grupo Investigación Didáctica.

- (2002). Acerca del origen y sentido de la educación inclusiva. Revista de Educación., 327, 11-29.

PARRILLA LATAS, A. y GALLEGO, C. (1999). Cómo pueden los profesores ayudarse entre sí: el desafío del trabajo compartido. Revista de Educación Especial, 25, 55-69.

PÉREZ GÓMEZ, A. I. y SOLA, M. (2003). Las contradicciones de la ESO. Evaluación externa en Andalucía. Cuadernos de Pedagogía, 320, 77-82.

PUJOLÀS, P. (1997). Intervenció psicopedagògica i assessorament curricular a l'Ensenyament Secundari Obligatori: I'atenció a la diversitat de necessitats educatives dels alumnes dins I'aula ordinària. Tesis Doctoral inédita. Universidad de Girona: Departamento de Pedagogía.

PUJOLÀS, P. (1999). Atención a la diversidad y aprendizaje cooperativo en la ESO. Revista de Educación Especial, 26, 43-97.

SLAVIN, R.E. y MADDEN, N.A. (1987). La integración en las clases ordinarias de los alumnos con retraso escolar. En MARTIN-MORENO, Q. Cuestiones sobre la organización del entorno del aprendizaje. Madrid: UNED, 207-225.

SUSINOS, T. (2002). Un recorrido por la inclusión educativa española. Investigaciones y experiencias más recientes. Revista de Educación, 327, 49-68.

TORRES, J. A. (2001). La atención a la diversidad y la formación permanente del profesorado de Educación Secundaria: Realidad y Necesidades. En BUENO, J. J., NÚÑ̃Z, T. e IGLESIAS, A. (eds). Atención educativa a la diversidad en el nuevo milenio. Atención educativa a la diversidad en el nuevo milenio. ( Jornadas de Universidades y Educación Especial. 18. A Coruña, Abril 2001). A Coruña: Universidade da Coruña, 523-538.

VILLA, R. A. y THOUSAND, J. S. (eds.) (1995). Creating an Inclusive School. Alexandria, Virginia: Association for Supervision and Curriculum Development. 